\title{
A Control Method for Balancing the SoC of Distributed Batteries in Islanded Converter-Interfaced Microgrids
}

\author{
Spyros I. Gkavanoudis, Konstantinos O. Oureilidis, \\ Georgios C. Kryonidis, and Charis S. Demoulias \\ Department of Electrical and Computer Engineering, Aristotle University, 54124 Thessaloniki, Greece \\ Correspondence should be addressed to Spyros I. Gkavanoudis; s.gkavan@gmail.com
}

Received 27 March 2016; Accepted 16 May 2016

Academic Editor: Francesco Profumo

Copyright (C) 2016 Spyros I. Gkavanoudis et al. This is an open access article distributed under the Creative Commons Attribution License, which permits unrestricted use, distribution, and reproduction in any medium, provided the original work is properly cited.

\begin{abstract}
In a low-voltage islanded microgrid powered by renewable energy sources, the energy storage systems (ESSs) are considered necessary, in order to maintain the power balance. Since a microgrid can be composed of several distributed ESSs (DESSs), a coordinated control of their state-of-charge (SoC) should be implemented, ensuring the prolonged lifespan. This paper proposes a new decentralized control method for balancing the SoC of DESSs in islanded microgrids, without physical communication. Each DESS injects a current distortion at $175 \mathrm{~Hz}$, when its SoC changes by $10 \%$. This distortion is recognized by every DESS, through a phase-locked loop (PLL). In order to distinguish the origin of the distortion, each DESS injects a distortion of different time duration. This intermediate frequency has been selected in order to avoid the concurrence with the usual harmonics. The DESSs take advantage of this information and inject a current proportional to the SoC. Implementing this strategy, a comparable number of charging/discharging cycles for each DESS are achieved. Furthermore, an active filter operation, implemented in the $d q$ rotating frame for each individual harmonic, is integrated in the control of the distributed generation units, supplying nonlinear loads with high-quality voltage. The effectiveness of this method is verified by detailed simulation results.
\end{abstract}

\section{Introduction}

The tremendous increase in renewable generation penetration level in the electrical grids has favored the development of the microgrid concept, which is defined as a cluster of interconnected distributed generation units (DGUs), loads, and ESSs [1]. A microgrid is able to operate in an uninterruptable way under grid-connected or islanded mode.

Different approaches concerning the effective energy management of such microgrids have been proposed in the literature. Most of them suggest a communication-based management system with central and local controllers [2,3]. However, if the communication is lost, the controllers may generate undesirable control commands. Therefore, dependence on communication reduces the reliability of the control strategy. On the other hand, decentralized methods without communication rely on the power sharing among the microsources by taking into account only local measurements. A control method without communication is the droop control method, where the DGUs emulate the operation of parallel synchronous generators [4].

In the technical literature, the critical role of the energy storage in microgrids is focused on the regulation of the voltage and frequency [5] and on preserving the power balance due to the intermittent operation of the renewable energy sources (RESs) [6]. Furthermore, other ancillary functions of the energy storage [7] may include the lowvoltage ride-through (LVRT) capability, load leveling, peak shaving, and operating reserve. Integration of ESSs in microgrids allows flexibility but adds further complexity in the energy management and control. A crucial parameter to be considered when designing the energy management control is the SoC of the ESS. The SoC is the ratio of the remaining capacity to its nominal one.

The conventional control methods do not take into account the different capacities of the DESSs, leading to a 
significant differentiation in their lifespans. Therefore, the applied power sharing methodology in an islanded microgrid should consider the SoC of each DESS as a crucial parameter. The target is to maintain a comparable number of charging/discharging cycles among all energy storage devices. Most of the technical references on SoC balancing refer to DC microgrids with distributed batteries [8,9]. In [10] a power sharing control for an autonomous AC microgrid with batteries is presented. Although the sharing is based on the battery SoC, extensive communication is proposed, using central and local controllers.

Another important issue of the islanded microgrid concerns the voltage quality. Since the common loads feature nonlinearity, the control of DGUs should incorporate strategies for the attenuation of the most common harmonics. Assuming only three-phase symmetrical loads, the harmonics of third order are absent. The literature deals with the harmonic problem by placing extra converters parallel to the nonlinear loads $[11,12]$ or incorporating the active filter operation in the internal control of the DGUs $[13,14]$. On the one hand, the placement of extra devices raises the initial investment of the microgrid, while it limits the further addition of nonlinear loads. On the other hand, the active filter control seems more effective. In [15], current harmonic loops are added in the current control, while in [16] the authors propose the distorted current waveform for the harmonics in phase with the voltage at the terminals of the DGU. In [17], an enhanced virtual impedance control on selected harmonics is implemented; however low-bandwidth communication is regarded. Other methodologies propose the use of resistive virtual impedances $[18,19]$. Nevertheless, the voltage is severely distorted. In [20], the control strategy employs negative virtual harmonic impedance, in order to compensate the effect of line impedance on harmonic power delivery. The harmonic attenuation methods proposed in the literature are implemented in either $a b c$ or $\alpha \beta$ stationary frame. In first case, independent control systems for each phase are required, provoking a more complex control scheme. In case the $\alpha \beta$ stationary frame is used, the resonant frequency of the proportional-resonant (PR) controllers should be updated for every frequency change.

This paper proposes a new decentralized method for controlling the SoC of DESSs in an islanded AC microgrid with linear and nonlinear loads. A primary aim of the control is to balance the number of charging/discharging cycles of the DESSs, by adjusting their power sharing. For this reason, a power sharing strategy according to the SoC of each DESS is implemented. This method is based on the superstition of a distortion at $175 \mathrm{~Hz}$ in the injected output current of each DESS, when the SoC changes by $10 \%$. In order to recognize the origin of the distortion, different time durations are used for each DESS. In this way, each DESS adapts the power injection according the SoC in respect to the SoC of the other DESSs. An intermediate frequency at $175 \mathrm{~Hz}$ has been selected in order to avoid the concurrence with harmonics of the basic frequency of $50 \mathrm{~Hz}$. Moreover, unlike the high frequency $(>3 \mathrm{kHz})$ power line communication (PLC) methods [21], where the signals might be attenuated by the low-pass filters, a signal at $175 \mathrm{~Hz}$ can effectively be propagated in the microgrid bus. The magnitude of the injected distortion has been selected in compliance with the permissible limits for harmonic distortion in the LV grids. The proposed method is integrated in the control of each DESS, without requiring any additional communication infrastructure leading in reduced installation cost. A second target of the proposed control is to deal with the nonlinear loads. A nonlinear method for attenuating the $5 \mathrm{th}, 7 \mathrm{th}$, 11th, and 13th harmonic of the voltage is applied. Unlike the presented methodologies in the literature, the proposed method is implemented in the $d q$ rotating frame for each individual harmonic order. Its effectiveness is highlighted in islanded microgrids, due to the operation with variable frequency around the preassigned limits, without needing any further control update.

The rest of the paper is structured as follows. In Section 2, the microgrid decentralized control strategy for power sharing and harmonic attenuation is presented in detail. In Section 3, the proposed control strategy is described, while in Section 4 analytical simulation tests validate the methodology in an islanded microgrid with linear and nonlinear loads, being fed by PVs and batteries.

\section{Microgrid Decentralized Control Strategy}

2.1. Droop Control Method. The microgrid can be consisted of different type of DGUs, which are interfaced to the microgrid via $\mathrm{DC} / \mathrm{AC}$ or $\mathrm{AC} / \mathrm{DC} / \mathrm{AC}$ converters. In order to avoid extra communication infrastructure, a decentralized strategy, based on the droop control method, is applied for the power sharing among the DGUs. Each DGU determines the frequency and the voltage magnitude of the common $\mathrm{AC}$ bus according to the injected active and reactive power, respectively. In this way, the DGUs emulate the parallel operation of synchronous generators. Considering inductive line impedances, the microgrid frequency $f$ is determined by the active power $P$, while the node voltage $V_{n}$ is determined by the reactive power $Q$, as it is described by the following equations:

$$
\begin{aligned}
f & =f^{*}-m \cdot P-m_{d} \frac{d P}{d t}, \\
V_{n} & =V^{*}-n \cdot Q-n_{d} \frac{d Q}{d t},
\end{aligned}
$$

where $f^{*}$ and $V^{*}$ are the output voltage frequency and magnitude at no load, $m$ and $n$ the droop coefficients, $P$ and $Q$ the average active and reactive power, and $m_{d}$ and $n_{d}$ the derivative droop coefficients, respectively. The active and reactive output power of the inverter can be adjusted by means of the droop coefficients, while the derivative terms absorb the abrupt variations, preserving the power sharing dynamic stability [22].

When the microgrid consists of RESs, the droop coefficients are adjusted in accordance with the available power 
$P_{\text {pot }}$. Thus, the droop coefficients are calculated by the following equations:

$$
\begin{gathered}
m=\frac{\Delta f_{\text {max }}}{P_{\text {pot }}}, \\
n=\frac{\Delta V_{\text {max }}}{P_{\text {pot }}},
\end{gathered}
$$

where $\Delta f_{\max }$ and $\Delta V_{\max }$ are the maximum deviation of the frequency of the magnitude of the voltage as it is defined by the Standard EN 50160.

However, in low-voltage microgrids the line impedance rarely is purely inductive. In most cases it consists of both resistive and inductive part [23]. With complex impedances, an accurate power sharing cannot be achieved, due to the coupled active and reactive power characteristic of the system. As a result, reactive currents are circulating among the DERs. To overcome this problem, the droop control method is modified with the virtual impedance control, as it is well stated in the literature [24]. The control scheme of each DGU is presented in Figure 1.

2.2. Harmonic Attenuation Control. The microgrid should be able to supply the loads with high-quality voltage, even under the presence of nonlinear loads. Therefore, an additional control is incorporated, in order to keep the voltage harmonic content within the permissible limits. In this paper, the microgrid consists of three-phase loads; for this reason the three-order harmonics are absent. Therefore, the proposed control deals with the attenuation of the most common harmonics, that is, the $5 \mathrm{th}, 7 \mathrm{th}, 11 \mathrm{th}$, and $13 \mathrm{th}$.

The structure of this control is based on the calculation of harmonic reactive power $H$ and its sharing among the sources. The reactive power sharing takes place according to [25]. This technique uses a $G-H$ droop to share the reactive harmonic power among the DGUs. The harmonic reactive power is calculated from the active and reactive power, as they are analyzed in average and alternating component:

$$
\begin{aligned}
P & =\bar{p}+\tilde{p}, \\
Q & =\bar{q}+\tilde{q}, \\
H & =\sqrt{\tilde{p}^{2}+\tilde{q}^{2}},
\end{aligned}
$$

where $p$ and $q$ are power measurements by means of Park Transformation, $\bar{p}$ and $\bar{q}$ are the mean values, and $\widetilde{p}$ and $\widetilde{q}$ are the alternating values. The sharing among the DGUs is carried out according to its apparent power, through the $G-H$ droop control as it is described in

$$
G=G_{0}-b H,
$$

where $G$ is the conductance of the DGU at harmonic frequencies, $G_{0}$ the conductance at no harmonic reactive power, and $b$ the droop coefficient. The maximum harmonic reactive power is rated at 0.65 times the apparent power, protecting the converter from large output currents. The last parameter $b$ is defined so as

$$
b_{1} H_{1}=b_{2} H_{2}=\cdots=b_{n} H_{n} .
$$

The harmonic control current is shown in Figure 2. According to this scheme, the measured voltage at the terminals of each DGU is transformed to the respective $d q$ rotating frame, implementing the Park Transformation for each harmonic. Therefore, the order and the sequence (positive or negative) of each harmonic are taken into consideration for the control angle. A similar control is also implemented for the current transformation. The philosophy of the harmonic attenuation control is to eliminate the distorted voltage at the selected harmonics. Thus, a voltage and current controller, similar to the conventional droop control of the basic frequency, is adopted. The voltage reference for each harmonic order is equal to zero, while the reference of the current is the output of the voltage controller.

2.3. DESS Control. The DESS control is embedded in the $\mathrm{DC} / \mathrm{AC}$ converter of the energy storage. Aim of the control is to measure the frequency and the magnitude of the common AC-bus voltage and determine the active and reactive power that should be absorbed or released, in order to ensure a highquality voltage. At least one DESS should be placed on the point of common connection with the utility grid, in order to synchronize the microgrid during the transition from the island to the grid-connected mode, avoiding the considerable transient effects [4]. This operation is not presented in this paper, since it is focused on the islanded operation of the microgrid.

The control of the DESS, using a battery as energy storage device, is described in Figure 3(a). The voltage magnitude and frequency are measured through a $d q$ phase-locked loop (PLL) [26], shown in Figure 3(b). When the frequency drops below a lower threshold $\left(f_{\min }=49 \mathrm{~Hz}\right)$, the DESS is connected releasing active power. The aim is to restore the frequency to the preassigned operating range (target frequency is set to 49.01), according to [27]. Control is performed through a closed loop employing PI controller:

$$
I_{d}=K_{p d 1}(49.01-f)+K_{i d 1} \int(49.01-f) d t .
$$

A similar control philosophy is adopted for defining the reactive power. When the voltage magnitude drops below a threshold $\left(V_{\min }=293 \mathrm{~V}\right)$, the converter of DESS injects reactive power. The reactive current signal is defined by

$$
I_{q}=K_{p q 1}\left(293-V_{n}\right)+K_{i q 1} \int\left(293-V_{n}\right) d t .
$$

If the DESS is not fully charged, and the microgrid frequency is higher than $50 \mathrm{~Hz}$ it charges with a current equal to $1 / 10$ of the nominal battery capacity.

The SoC of the DESS is constantly measured, as

$$
\text { SoC }=\frac{Q_{o}-\int i_{\text {bat }} d t}{Q_{\text {tot }}},
$$

where $Q_{o}$ is the initial charge of the battery, $Q_{\text {tot }}$ the maximum charge of the battery, and $i_{\text {bat }}$ the output current of the battery. The SoC limits for the usual charge-discharge cycle, ensuring an extended lifetime, are considered among $20 \%$ and $90 \%$. 


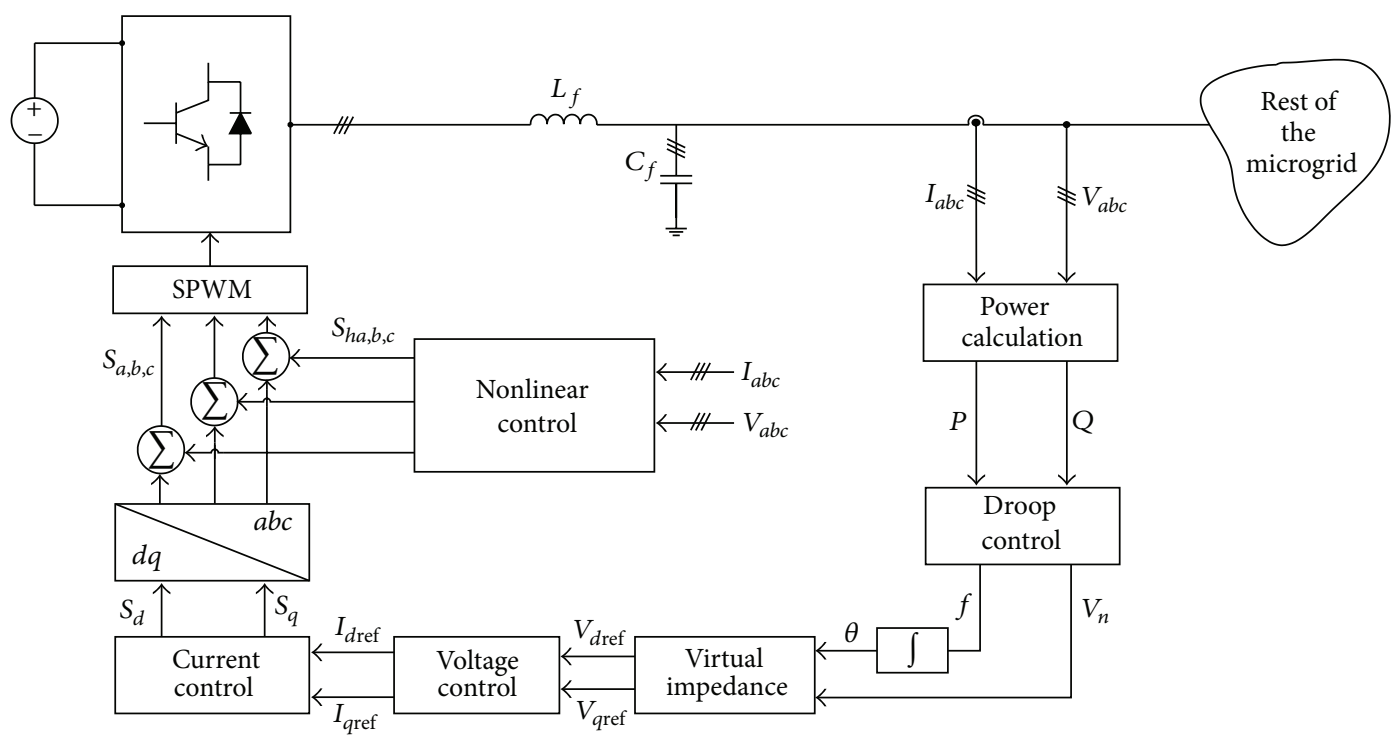

FIGURE 1: DGU converter control strategy.

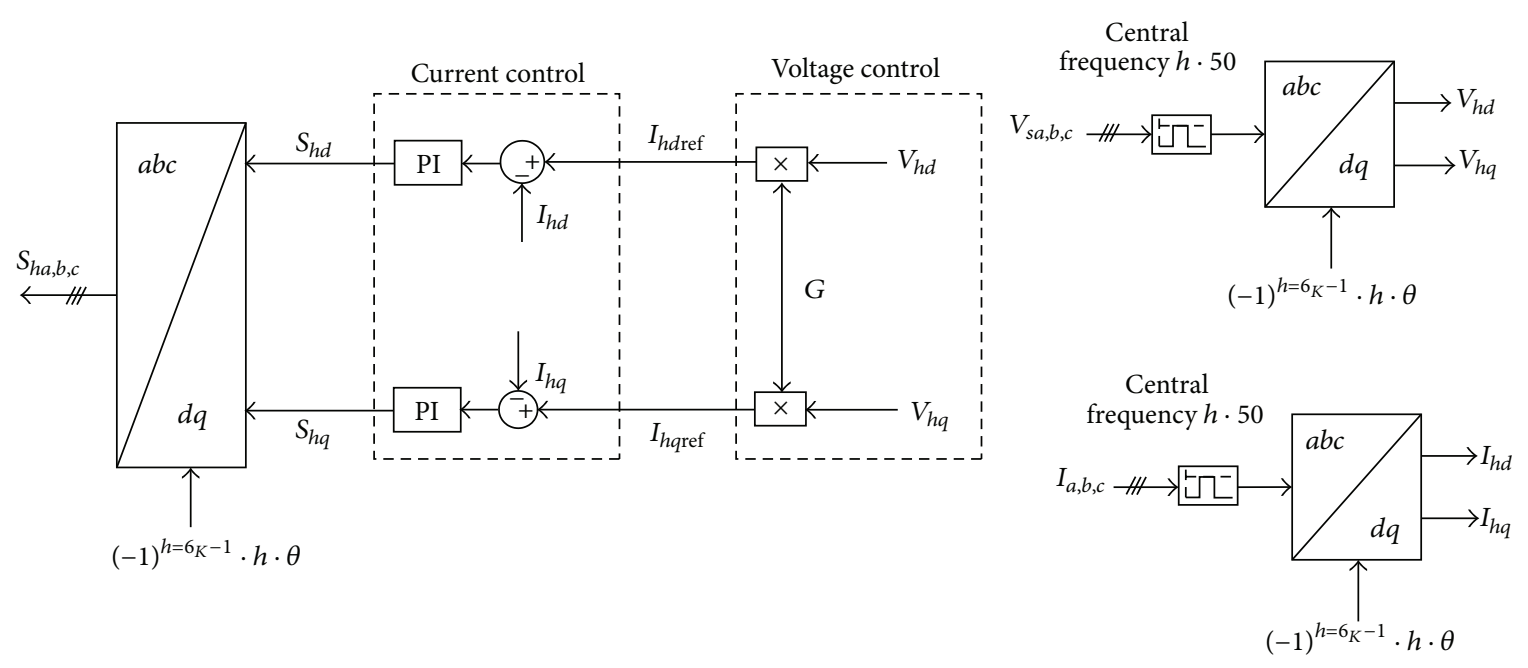

FIgURE 2: Nonlinear control.

The reference for the active and reactive current injected by each DESS is modified by the ratio of the respective SoC to the aggregated SoC levels of all DESSs. In this way, the sharing of the active and reactive power demand among the DERs is performed proportional to their SoC. The active $\left(I_{d \text { ref }}\right)$ and reactive $\left(I_{\text {qref }}\right)$ current reference signals are given by

$$
I_{d q \mathrm{ref}, i}=I_{d q, i} \cdot \frac{\mathrm{SoC}_{i}}{\sum_{j=1}^{N} \mathrm{SoC}_{j}},
$$

where $\mathrm{SoC}_{i}$ is the SoC of the $i$-battery and $N$ is the amount of the connected batteries. In order to ensure that the SoC will always remain within the predefined limits (20\%-90\%), once it reaches the lower limit, no more energy is released. Respectively, when the SoC of the battery reaches the upper limit, no more energy is absorbed. In this way, the SoC cannot exceed either the upper or the lower limit. Similar to the control of the DGU, the SPWM method is used in the output of the current controller, as shown in Figure 3(a).

\section{Battery Management Control Strategy}

3.1. Key Principles of the Proposed Method. In order to ensure that each battery of the microgrid will have comparable number of charging/discharging cycles, the SoC should be effectively controlled. The SoC range is divided into equal levels, each representing $10 \%$ of the charge capacity. The proposed method is based on the superimposition of a sinusoidal distortion at $175 \mathrm{~Hz}$ in the injected output current of each DESS, when the SoC changes by $10 \%$. More specifically, two distortions with different time durations are implemented in order to represent the $10 \%$ increase and $10 \%$ decrease of the SoC, respectively. Furthermore, in order to distinguish the origin of the distortion, each DESS uses different time 


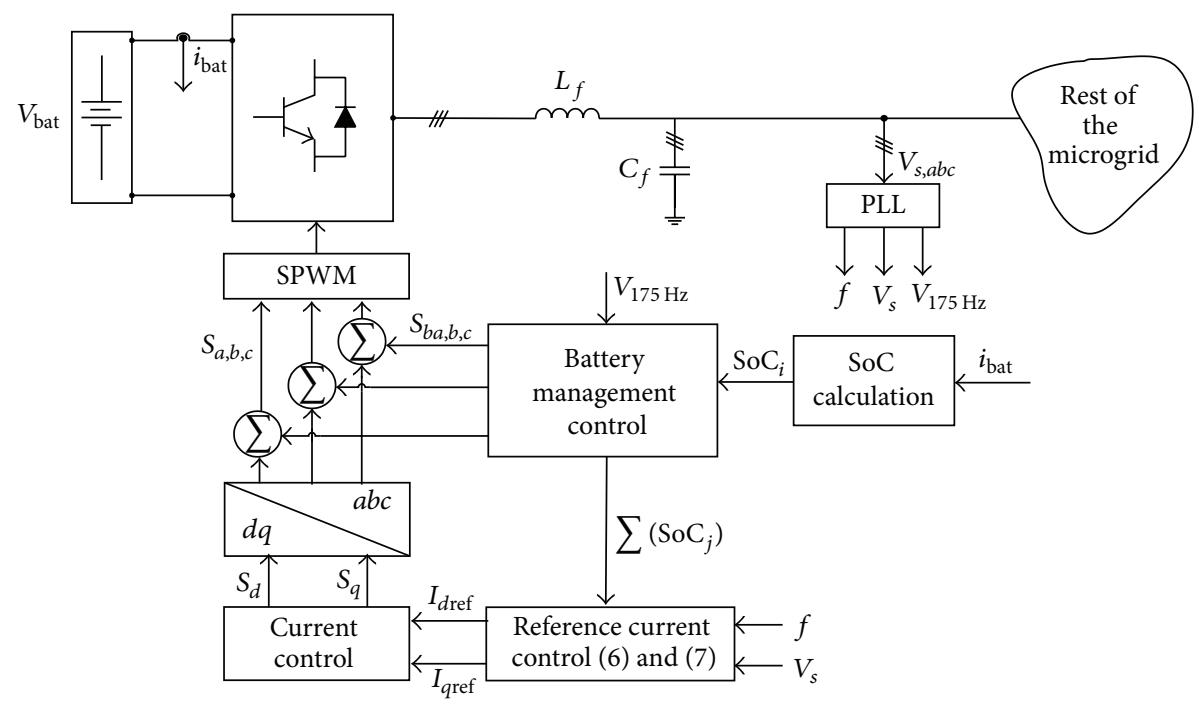

(a)

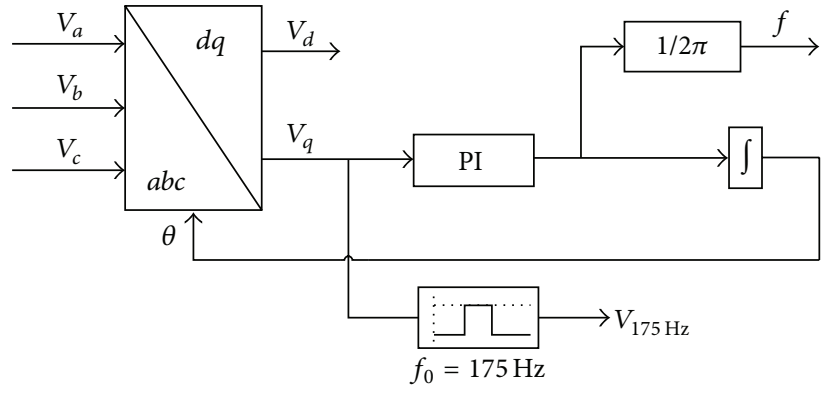

(b)

FIGURE 3: (a) DESS converter control strategy and (b) $d q$-PLL.

durations. The time duration of each injected distortion is described by an integer defining the number of periods $(T=$ $1 / 175 \mathrm{sec}$ ). Two unique integers are assigned to each DESS, in order to differentiate the SoC increase from the SoC decrease. Therefore, by counting the number of periods, the origin of the distortion signal can be classified to the corresponding DESS.

Beginning from a black start, the SoC level of a DESS is considered to be zero. Therefore, the DESS will send a number of sequential current distortions, each representing an increase of $10 \%$ in the SoC (e.g., if the SoC is $70 \%$, seven sequential distortions will be transmitted). Another issue concerns the conflict among the simultaneous transmissions. For this reason, a time delay is also inserted. Thus, when a DESS identifies a distortion, the transmission control is deactivated.

In order to implement the proposed methodology, the internal control strategy of the DESS is modified. The SoC increase or decrease information is transmitted in form of a sinusoidal distortion at $175 \mathrm{~Hz}$, superimposed in the output current. The parameters of this distortion, such as the amplitude and frequency, are the same regardless of its origin. The information is contained on its time duration (number of periods).
3.2. Distortion Signal Characteristics. The frequency of the distortion is selected at $175 \mathrm{~Hz}$, which is an intermediate frequency, in order to prevent any interference with the harmonics of the basic frequency $50 \mathrm{~Hz}$, that is, $250 \mathrm{~Hz}$, $350 \mathrm{~Hz}$, and so forth. Moreover, the selection of a high frequency has been avoided, in order not to be attenuated by the low-pass filters.

The Standard EN 50160 does not define the exact permissible amplitude for the intermediate frequency signals. However, it specifies the maximum allowable amplitude of the communication signals, as shown in Figure 4. Around the frequency of $175 \mathrm{~Hz}$, the upper limit for the amplitude is $9 \%$ of the maximum nominal grid phase voltage. For this reason, the amplitude of the distortion is selected at $1 \%$, that is, $3.2 \mathrm{~V}$. This value is rather small, in order not to encumber the microgrid bus with additional harmonics.

3.3. Method Implementation. The implementation of the proposed method requires a transmitter and receiver for the distortion in each DESS, implemented in the battery management control block. The battery management control is placed before the PWM control of the DESS converter (Figure 3). Figure 5(a) presents the control of the transmitter, placed before the SPWM control. This control is divided into 


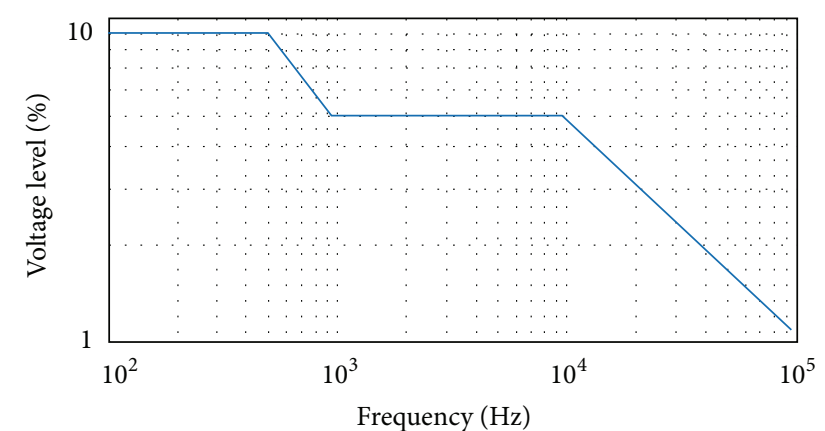

FIGURE 4: Allowable amplitude of intermediate harmonic for communication.

4 steps: the SoC control, the delay control, the reference signal control, and the resonant controller.

The first step (SoC control) is activated, when the SoC level of the battery varies by $10 \%$ (charging or discharging). Then, a sinusoidal reference signal of $3.2 \mathrm{~V}$ magnitude and $175 \mathrm{~Hz}$ frequency is produced. In case the transmitter has detected a distortion signal from another DESS, the delay control is activated to prevent conflicts raised by the simultaneous transmission of two or more distortion signals. Finally, the R-controller is responsible for the signal superimposition at each phase of the system. In the meantime, the transmitter counter block counts the periods of the output signal and deactivates the SoC transmitter control, when the signal reaches the predefined time duration. The transfer function of the R-controller is

$$
H(s)=\frac{2 k_{i} \omega_{c} s}{s^{2}+2 \omega_{c} s+\omega^{2}},
$$

where $\omega_{c}$ is considered within $5-15 \mathrm{rad} / \mathrm{s}, \omega$ is the resonance angular frequency, and $k_{i}$ is a gain.

The receiver control is divided into 2 steps, as presented in Figure 5(b). Initially, the distortion is detected and its time duration is measured, by using the $d q$-PLL in each DESS, as presented in Figure 3. The inputs of the PLL are the phase voltages at the terminals of the DESS. The output of the PLL in the $q$-axis $\left(V_{q}\right)$ is fed in a band-pass filter, that is tuned at $175 \mathrm{~Hz}$, in order to isolate the distortion. The bandwidth of the band-pass filter is selected equal to $20 \mathrm{~Hz}$. In this way, the distortion signal is extracted from the voltage waveform. The time duration of the distortion is measured in the receiver counter block, by counting the number of its periods. In the next step, the origin of the distortion is identified in the identification block, by comparing the time duration of the distortion with the predefined number of periods corresponding to each DESS.

\section{Simulation Tests}

4.1. Simulation Model. The microgrid consists of two converter-interfaced DGUs, three DESSs with DC-batteries, a constant power linear load, and two nonlinear loads $\left(\mathrm{NL}_{1}\right.$ and $\mathrm{NL}_{2}$ ), as it is shown in Figure 6. The parameters of the system are listed in Table 1, while the electrical parameters
TABLE 1: System parameters.

\begin{tabular}{lcc}
\hline Items & $\mathrm{DGU}_{1}$ & $\mathrm{DGU}_{2}$ \\
\hline Rated power $S(\mathrm{kVA})$ & 10 & 5 \\
Frequency droop coefficient $m$ & $2 \cdot 10^{-4}$ & $4 \cdot 10^{-4}$ \\
Derivative frequency coefficient $m_{d}$ & $10^{-5}$ & $10^{-5}$ \\
Amplitude droop coefficient $n$ & $3.25 \cdot 10^{-3}$ & $6.5 \cdot 10^{-3}$ \\
Derivative amplitude coefficients $m_{d}, n_{d}$ & $10^{-5}$ & $10^{-5}$ \\
Virtual inductor $L_{o}(\mathrm{mH})$ & 4 & 10 \\
\hline
\end{tabular}

TABLE 2: Transmitter/receiver characteristics.

\begin{tabular}{lccc}
\hline $\begin{array}{l}\text { Battery } \\
\text { (number) }\end{array}$ & $\begin{array}{c}\text { Initial SoC } \\
(\%)\end{array}$ & $\begin{array}{c}10 \% \text { SoC increase } \\
\text { (number of } \\
\text { periods) }\end{array}$ & $\begin{array}{c}10 \% \text { SoC decrease } \\
\text { (number of } \\
\text { periods) }\end{array}$ \\
\hline 1 & 80 & 7 & 10 \\
2 & 40 & 13 & 16 \\
3 & 60 & 19 & 22 \\
\hline
\end{tabular}

of the connection line are overhead ACSR $16 \mathrm{~mm}^{2}$ lines with $R=1.268 \Omega / \mathrm{km}$ and $X=0.422 \Omega / \mathrm{km}$. The length of each connection line is $L_{1}=100 \mathrm{~m}, L_{2}=200 \mathrm{~m}$, $L_{3}=150 \mathrm{~m}, L_{4}=300 \mathrm{~m}, L_{5}=100 \mathrm{~m}$, and $L_{6}=50 \mathrm{~m}$. Each battery converter has $5 \mathrm{kVA}$ nominal apparent power and is composed of $400 \mathrm{OPzS}$-type lead-acid batteries of $2 \mathrm{~V}$ nominal voltage. The battery bank is modelled as a variable DC voltage source in series with internal resistance [28]. The switching frequency of all converters is $9.95 \mathrm{kHz}$, while the DC voltage of all DGUs is $800 \mathrm{~V}$. All the simulation tests are carried out in the time domain with the PSIM Software.

The characteristics of each battery management controller are presented in Table 2. Each DESS owns two uniqueduration signals: one for the state of $10 \%$ increase in SoC and the other for $10 \%$ decrease.

\subsection{Simulation Results}

4.2.1. Case 1: Power Sharing (with Linear Load Increase). Initially, the power combination of the two DGUs feeds the linear and nonlinear load with active power of $7 \mathrm{~kW}$ and $4 \mathrm{~kW}$, respectively. At $t=1 \mathrm{~s}$ the linear load increases by $7 \mathrm{~kW}$. The DGUs adapt the injected active power trying to fulfill the load demand, according to the droop control method. As a result, the frequency drops below $49 \mathrm{~Hz}$, since the aggregated load demand is larger than the aggregated available power of the two DGUs. The battery converters measure a frequency lower than $49 \mathrm{~Hz}$ and are connected to the microgrid. The active power sharing among the batteries is performed proportional to their SoC, according to the proposed strategy, described in Section 2.3. Therefore, the frequency is restored above the acceptable limit of $49 \mathrm{~Hz}$. Figure 7 (a) presents the power sharing of the two DGUs, while Figure 7(b) presents the formed microgrid frequency. The active power injected by each DESS is presented in Figure 7(c), while the respective SoC is presented in Figure $7(\mathrm{~d})$. As it is obvious, Bat 2 has the lowest SoC and consequently injects less power than the other two batteries. At $t=5.2 \mathrm{~s}$, the SoC of Bat_2 becomes 


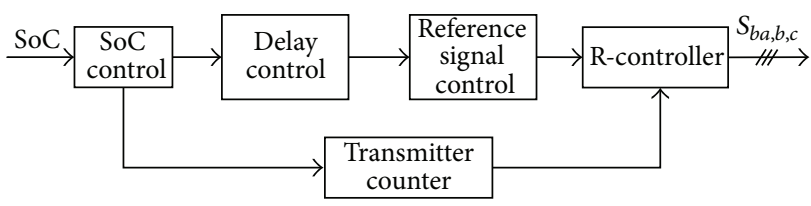

(a)

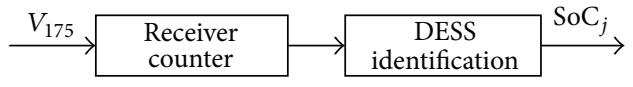

(b)

FIGURE 5: Battery management control: (a) SoC transmitter control and (b) SoC receiver control.

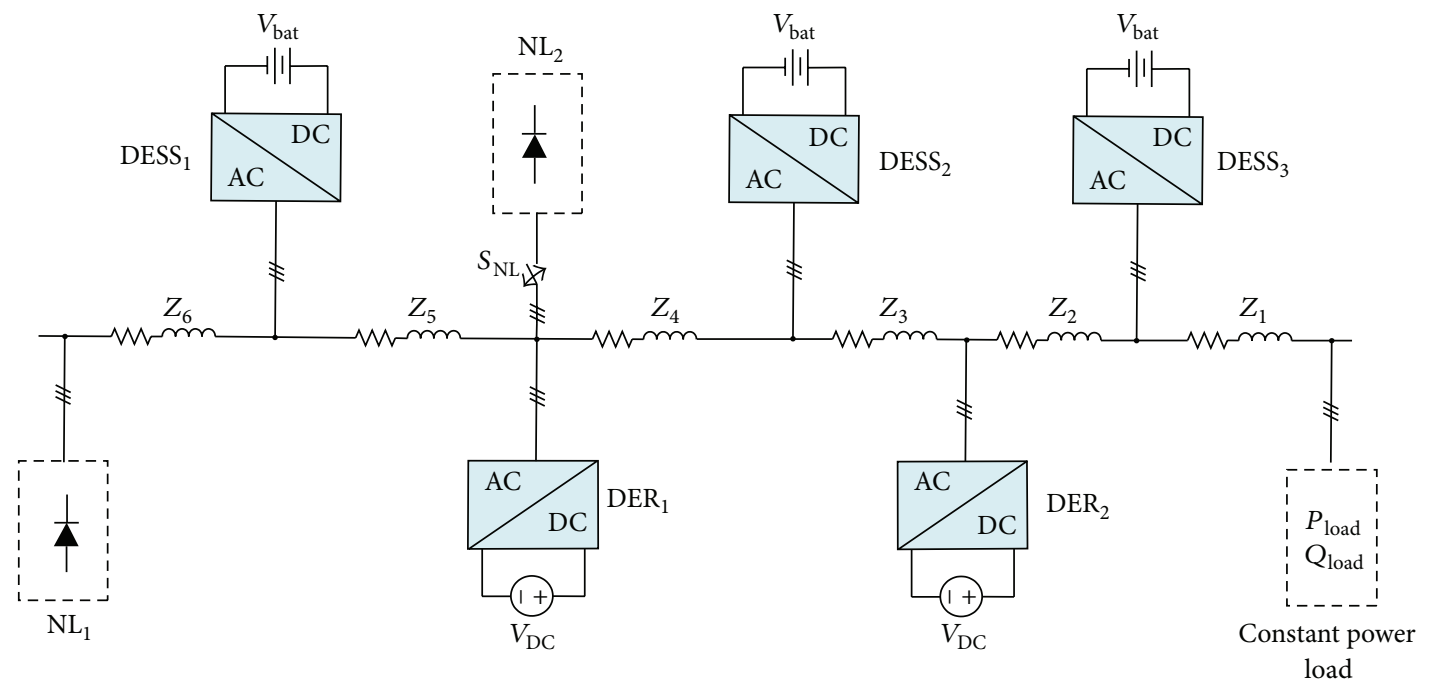

Figure 6: Microgrid scheme.

$20 \%$ (which is considered the lowest permissible limits). As a result, its active power injection is zeroed, as can be seen in Figure 7(c). The other two DESSs undertake the load demand, in order to maintain the frequency within the limits defined by EN 50160. Evaluating this method, a comparable number of charging/discharging cycles for all batteries will be achieved and at the meantime they are protected from deep discharges. In case the proposed SoC control was not applied, each DESS would inject the same amount of active power, leading to the depletion of the battery with the lowest SoC.

In order to perform the power sharing according to the SoC, each DESS integrates a battery management control. The control is implemented by injecting a distortion at $175 \mathrm{~Hz}$ each time the SoC decreases by $10 \%$. In Figure 8(a), the number of periods of the distortion signal transmitted by each DESS is presented, which is in accordance with Table 2. When the batteries are feeding the microgrid with active power, their SoC is reduced, as they discharge. At $t=2.6 \mathrm{~s}$, the SoC of battery Bat_2 becomes 30\%. This means that its SoC is reduced by $10 \%$ in respect to the initial SoC (Table 2) and as a result a distortion signal with 16-period time duration is propagated (blue curve in Figure 8(a)). This distortion signal is identified by all DESSs, as it is indicatively shown for Bat_1 in Figure 8(b). In a respective manner, distortion signals with different number of periods for each DESS are generated, transmitting the information of their SoC variation. According to the counter measurements, each DESS can identify the origin of the distortion and adjust the initial SoC. Figure 8(c) presents the SoC for each battery as they are perceived by Bat_1. By implementing the proposed battery management control each DESS can effectively identify the SoC of the other batteries without implementing physical communication.

4.2.2. Case 2: Power Sharing (with Load Increase and Decrease). In this test case, the proposed power sharing method is examined under varying load conditions. Initially, the linear load demand is $7 \mathrm{~kW}$, while the nonlinear load demand is $4 \mathrm{~kW}$. At $t=1 \mathrm{~s}$, the linear load increases by $7 \mathrm{~kW}$ and the microgrid frequency decreases below $49 \mathrm{~Hz}$ (Figure $9(\mathrm{~b})$ ), since the DGUs supply their maximum available active power (Figure 9(a)). Therefore, the DESSs inject active power in order to satisfy the load demand and restore the frequency within the permissible limits. At $t=3 \mathrm{~s}$, the linear load is decreased by $3 \mathrm{~kW}$. The DESSs contribute to less active power, as can be seen in Figure $9(\mathrm{c})$. This is reflected in the rate of SoC decrease of each battery (Figure 9(d)). A successive load increase of $2 \mathrm{~kW}$ takes place at $t=6 \mathrm{~s}$. The DESSs increase again their active power injection in order to control the microgrid frequency. The power sharing among the DESSs is performed proportional to their SoC, proving the effectiveness of the proposed method, even under load variations.

4.2.3. Case 3: Harmonic Attenuation. In an islanded microgrid with both linear and nonlinear loads, the voltage is polluted with harmonics of higher order. This test case demonstrates the effectiveness of the proposed voltage harmonic 


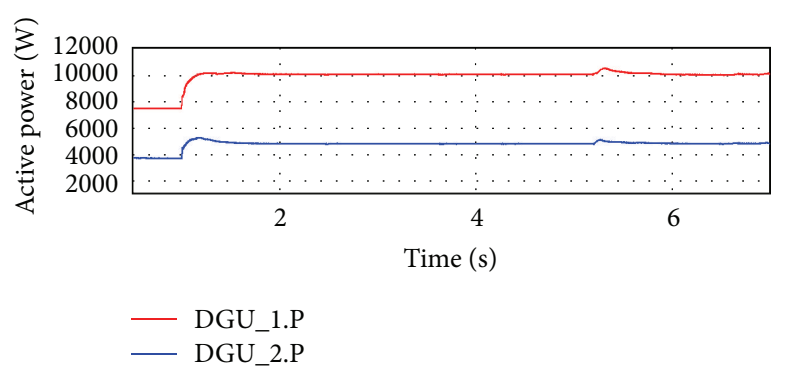

(a)

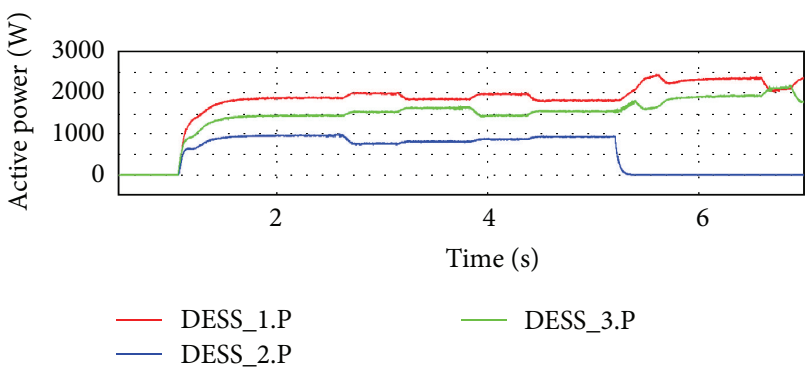

(c)

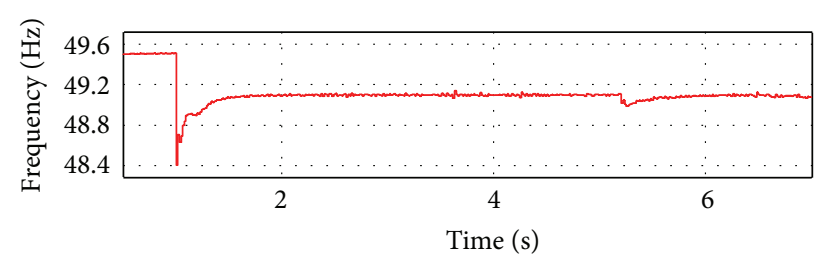

- Frequency

(b)

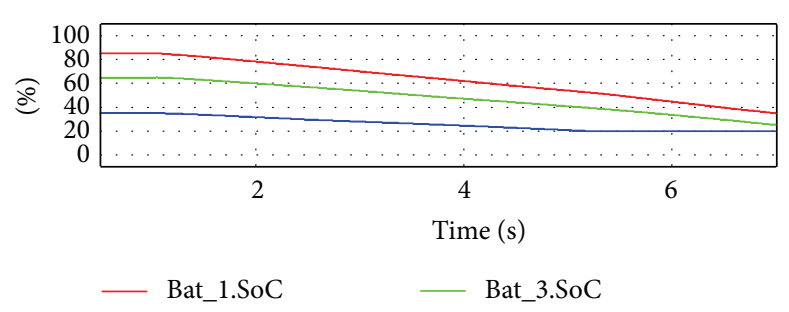

(d)

FIGURE 7: (a) DGU active power injection, (b) microgrid frequency, (c) DESS active power injection, and (d) battery actual SoC.

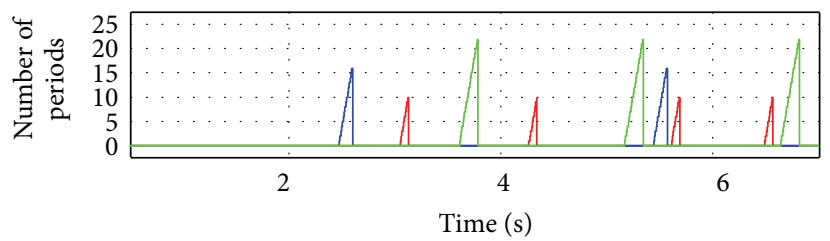

— DESS_1.counter transmit — DESS_3.counter transmit —_DESS_2.counter transmit

(a)

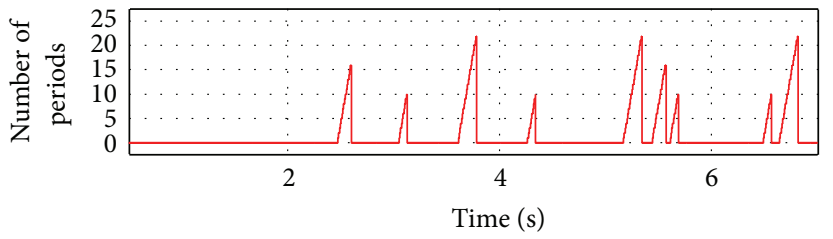

— DESS_1.counter receiver

(b)

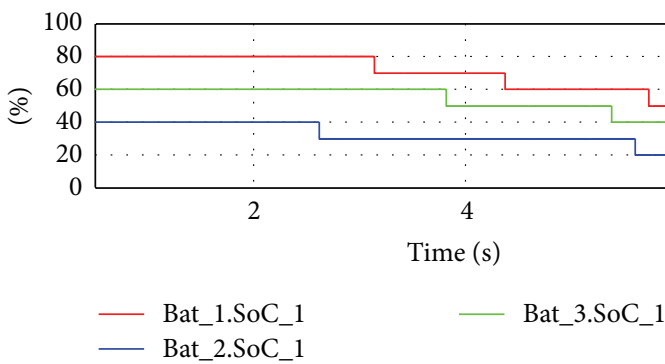

(c)

FIGURE 8: (a) Number of periods of distortion signal transmitted, (b) number of periods of distortion signal as measured by the receiver counter, and (c) battery SoC measured by DESS_l.

attenuation method under increase of the nonlinear active power absorption. The increase of the active power implies the change in the microgrid frequency. In the following simulations scenarios, the nonlinear load is simulated as a three-phase diode rectifier with resistance. Initially, the linear load and the nonlinear load $\mathrm{NL}_{1}$ are connected to the microgrid, as it is shown in Figure 6. The active power absorption is $7 \mathrm{~kW}$ for the linear load and $4 \mathrm{~kW}$ for $\mathrm{NL}_{1}$. The microgrid operates with frequency equal to $49.52 \mathrm{~Hz}$.
At $t=0.5 \mathrm{~s}$, the switch $\mathrm{sw}_{\mathrm{NL}}$ is activated and the nonlinear load $\mathrm{NL}_{2}$ is connected, adding $9 \mathrm{~kW}$ of active power. As a result, the frequency changes to $49.09 \mathrm{~Hz}$. Figures 10(a) and $10(\mathrm{~b})$ present the phase voltage profile (phase $a$ ) at the terminals of the linear load without the action of the proposed harmonic attenuation method. In this case, the total harmonic distortion $\left(\mathrm{THD}_{v}\right.$ ) is equal to $5.3 \%$ (only $\mathrm{NL}_{1}$ is connected) and $8.12 \%$ (both $\mathrm{NL}_{1}$ and $\mathrm{NL}_{2}$ are connected), respectively. The same simulation test is performed after the 


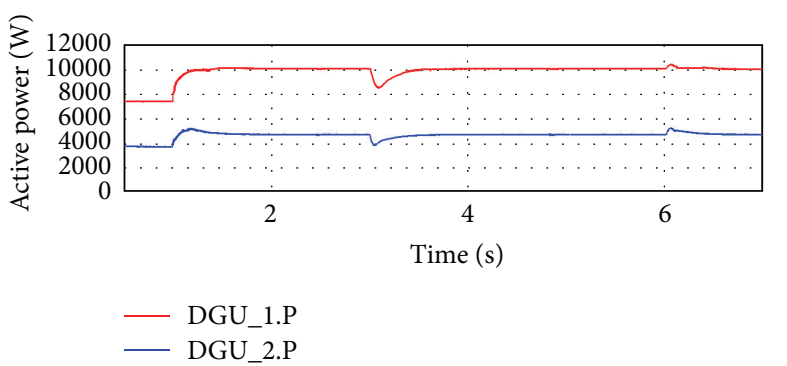

(a)

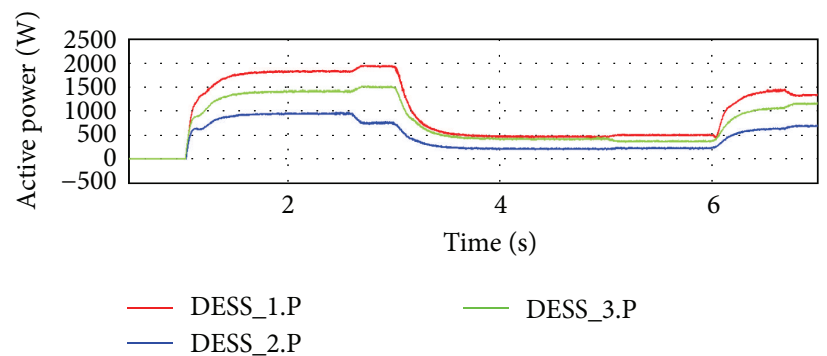

(c)

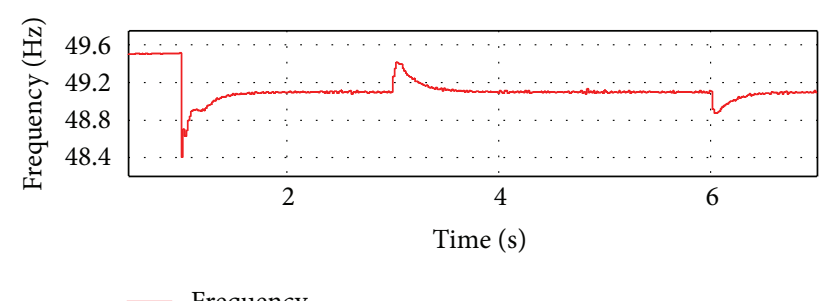

(b)

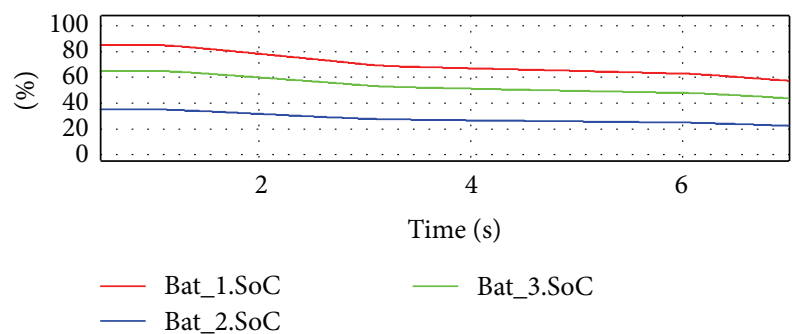

(d)

FIGURE 9: (a) DGU active power injection, (b) microgrid frequency, (c) DESS active power injection, and (d) battery actual SoC.

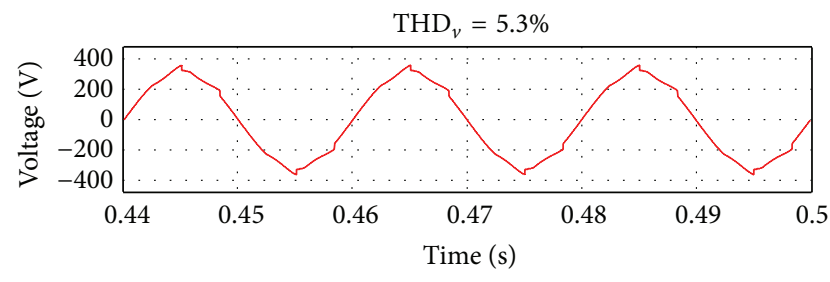

$-V_{a}$

(a)

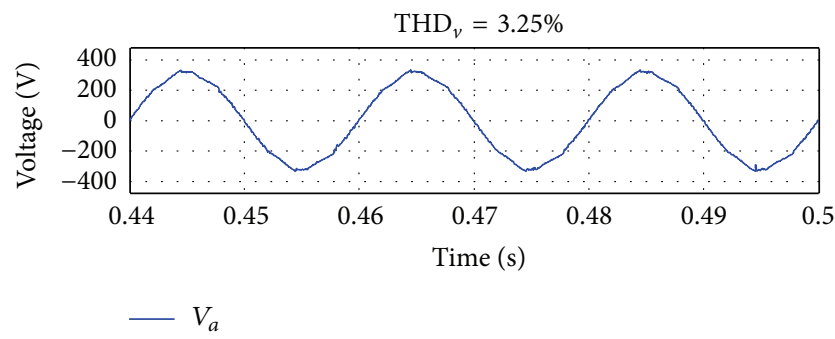

(c)

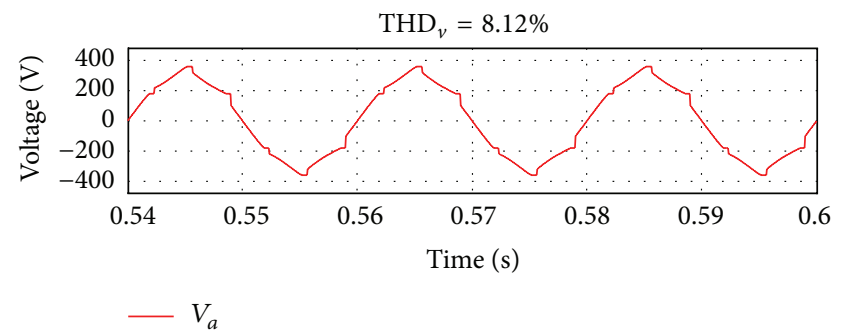

(b)

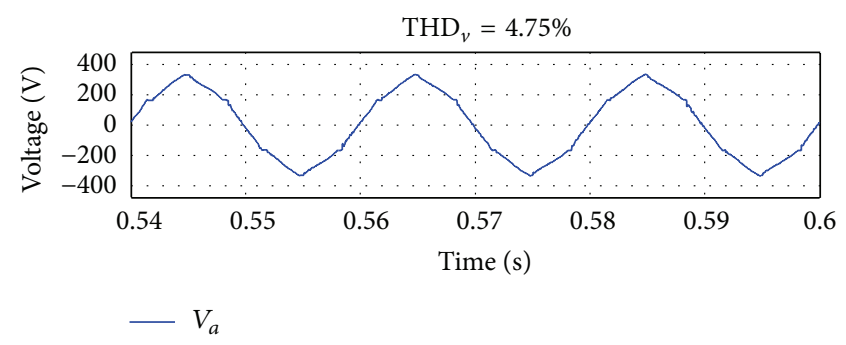

(d)

FIGURE 10: Voltage (phase $a$ ) at the terminals of the linear load: (a) with $\mathrm{NL}_{1}$ connected and deactivated the proposed control, (b) with $\mathrm{NL}_{1}$ and $\mathrm{NL}_{2}$ connected and deactivated the proposed control, (c) with $\mathrm{NL}_{1}$ connected and activated the proposed control, and (d) with $\mathrm{NL}_{1}$ and $\mathrm{NL}_{2}$ connected and activated the proposed control.

activation of the proposed control method. As can be noticed from Figures $10(\mathrm{c})$ and $10(\mathrm{~d}), \mathrm{THD}_{v}$ decreases significantly to $3.25 \%$ and $4.75 \%$, respectively. The effectiveness of the proposed method is verified even under severe nonlinear load and frequency deviations.

\section{Conclusions}

In an islanded microgrid with several DESSs, the SoC balancing problem should be effectively addressed, in order to ensure a prolonged lifespan. In contrary to communicationbased techniques, this paper proposes a new decentralized method in order to define the injected current of each DESS. According to this methodology, a sinusoidal distortion at $175 \mathrm{~Hz}$ is superimposed in the output current of every DESS and propagated in the microgrid bus. This distortion indicates the increase or decrease of the SoC. In this way, the information of the SoC is indirectly communicated to the other DESSs, which adapt their internal control and regulate the power sharing proportional to their SoC. The target is 
to achieve a comparable number of charging/discharging cycles. The proposed method is integrated in the control of each DESS, without requiring any additional communication infrastructure leading to reduced installation cost. Since the islanded microgrid may also contain nonlinear loads, an effective harmonic attenuation method is also proposed. This method is applied to each DGU and is implemented in the $d q$ rotating frame. Thus, the harmonic attenuation control is immune to frequency deviations. The simulation results show the effectiveness of the proposed control method, in an islanded microgrid with several DGUs and batteries as DESS.

\section{Competing Interests}

The authors declare that they have no competing interests.

\section{References}

[1] N. Hatziargyriou, H. Asano, R. Iravani, and C. Marnay, "Microgrids," IEEE Power and Energy Magazine, vol. 5, no. 4, pp. 78-94, 2007.

[2] J.-Y. Kim, J.-H. Jeon, S.-K. Kim et al., "Cooperative control strategy of energy storage system and microsources for stabilizing the microgrid during islanded operation," IEEE Transactions on Power Electronics, vol. 25, no. 12, pp. 3037-3048, 2010.

[3] K. T. Tan, X. Y. Peng, P. L. So, Y. C. Chu, and M. Z. Q. Chen, "Centralized control for parallel operation of distributed generation inverters in microgrids," IEEE Transactions on Smart Grid, vol. 3, no. 4, pp. 1977-1987, 2012.

[4] K. O. Oureilidis and C. S. Demoulias, "An enhanced role for an energy storage system in a microgrid with converter-interfaced sources," The Journal of Engineering, 2014.

[5] S. Koohi-Kamali, V. V. Tyagi, N. A. Rahim, N. L. Panwar, and H. Mokhlis, "Emergence of energy storage technologies as the solution for reliable operation of smart power systems: a review," Renewable and Sustainable Energy Reviews, vol. 25, pp. 135-165, 2013.

[6] J.-Y. Kim, J.-H. Jeon, S.-K. Kim et al., "Cooperative control strategy of energy storage system and microsources for stabilizing the microgrid during islanded operation," IEEE Transactions on Power Electronics, vol. 25, no. 12, pp. 3037-3048, 2010.

[7] X. Tan, Q. Li, and H. Wang, "Advances and trends of energy storage technology in Microgrid," International Journal of Electrical Power \& Energy Systems, vol. 44, no. 1, pp. 179-191, 2013.

[8] X. Lu, K. Sun, J. M. Guerrero, J. C. Vasquez, and L. Huang, "State-of-charge balance using adaptive droop control for distributed energy storage systems in DC microgrid applications," IEEE Transactions on Industrial Electronics, vol. 61, no. 6, pp. 2804-2815, 2014.

[9] C. Li, T. Dragicevic, N. L. Diaz, J. C. Vasquez, and J. M. Guerrero, "Voltage scheduling droop control for State-of-Charge balance of distributed energy storage in DC microgrids," in Proceedings of the IEEE International Energy Conference (ENERGYCON '14), pp. 1310-1314, Cavtat, Croatia, May 2014.

[10] O. Palizban and K. Kauhaniemi, "Power sharing for distributed energy storage systems in AC microgrid: based on state-ofcharge," in Proceedings of the IEEE PES Asia-Pacific Power and Energy Engineering Conference (APPEEC '15), pp. 1-5, Brisbane, Australia, November 2015.
[11] Y. Li, D. M. Vilathgamuwa, and P. C. Loh, "Microgrid power quality enhancement using a three-phase four-wire gridinterfacing compensator," IEEE Transactions on Industry Applications, vol. 41, no. 6, pp. 1707-1719, 2005.

[12] L. Yun Wei, D. M. Vilathgamuwa, and L. Poh Chiang, "A grid-interfacing power quality compensator for three-phase three-wire microgrid applications," IEEE Transactions on Power Electronics, vol. 21, pp. 1021-1031, 2006.

[13] N. Pogaku and T. C. Green, "Application of inverter-based distributed generators for harmonic damping throughout a distribution network," in Proceedings of the IEEE 36th Power Electronics Specialists Conference (PESC '05), pp. 1922-1927, 2005.

[14] N. Pogaku and T. C. Green, "Harmonic mitigation throughout a distribution system: a distributed-generator-based solution," IEE Proceedings-Generation, Transmission and Distribution, vol. 153, no. 3, pp. 350-358, 2006.

[15] J. M. Guerrero, J. Matas, L. Garcia De Vicunagarcia De Vicuna, M. Castilla, and J. Miret, "Wireless-control strategy for parallel operation of distributed-generation inverters," IEEE Transactions on Industrial Electronics, vol. 53, no. 5, pp. 1461-1470, 2006.

[16] T. Takeshita and N. Matsui, "Current waveform control of PWM converter system for harmonic suppression on distribution system," IEEE Transactions on Industrial Electronics, vol. 50, no. 6, pp. 1134-1139, 2003.

[17] J. He, Y. W. Li, J. M. Guerrero, F. Blaabjerg, and J. C. Vasquez, "An islanding Microgrid power sharing approach using enhanced virtual impedance control scheme," IEEE Transactions on Power Electronics, vol. 28, no. 11, pp. 5272-5282, 2013.

[18] M. Savaghebi, J. M. Guerrero, A. Jalilian, J. C. Vasquez, and T.-L. Lee, "Hierarchical control scheme for voltage harmonics compensation in an islanded droop-controlled microgrid," in Proceedings of the IEEE 9th International Conference on Power Electronics and Drive Systems (PEDS '11), pp. 89-94, Singapore, December 2011.

[19] T. Vandoorn, B. Meersman, J. De Kooning, and L. Vandevelde, "Controllable harmonic current sharing in islanded microgrids: DG units with programmable resistive behavior toward harmonics," IEEE Transactions on Power Delivery, vol. 27, no. 2, pp. 831-841, 2012.

[20] P. Sreekumar and V. Khadkikar, "A new virtual harmonic impedance scheme for harmonic power sharing in an islanded microgrid," IEEE Transactions on Power Delivery, vol. 31, no. 3, pp. 936-945, 2015.

[21] A. N. Milioudis, K. N. Syranidis, G. T. Andreou, and D. P. Labridis, "Modeling of medium-voltage power-line communication systems noise levels," IEEE Transactions on Power Delivery, vol. 28, no. 4, pp. 2004-2013, 2013.

[22] J. M. Guerrero, M. Chandorkar, T.-L. Lee, and P. C. Loh, "Advanced control architectures for intelligent microgridspart I: decentralized and hierarchical control," IEEE Transactions on Industrial Electronics, vol. 60, no. 4, pp. 1254-1262, 2013.

[23] W. Yao, M. Chen, J. Matas, J. M. Guerrero, and Z.-M. Qian, "Design and analysis of the droop control method for parallel inverters considering the impact of the complex impedance on the power sharing," IEEE Transactions on Industrial Electronics, vol. 58, no. 2, pp. 576-588, 2011.

[24] H. Mahmood, D. Michaelson, and J. Jiang, "Accurate reactive power sharing in an islanded microgrid using adaptive virtual impedances," IEEE Transactions on Power Electronics, vol. 30, no. 3, pp. 1605-1617, 2015. 
[25] T.-L. Lee and P.-T. Cheng, "Design of a new cooperative harmonic filtering strategy for distributed generation interface converters in an islanding network," IEEE Transactions on Power Electronics, vol. 22, no. 5, pp. 1919-1927, 2007.

[26] P. Rodríguez, J. Pou, J. Bergas, J. I. Candela, R. P. Burgos, and D. Boroyevich, "Decoupled double synchronous reference frame PLL for power converters control," IEEE Transactions on Power Electronics, vol. 22, no. 2, pp. 584-592, 2007.

[27] European Standard, "Voltage characteristics of electricity supplied by public distribution system," Standard EN 50160, 2004.

[28] K. O. Oureilidis, E. A. Bakirtzis, and C. S. Demoulias, "Frequency-based control of islanded microgrid with renewable energy sources and energy storage," Journal of Modern Power Systems and Clean Energy, vol. 4, no. 1, pp. 54-62, 2016. 


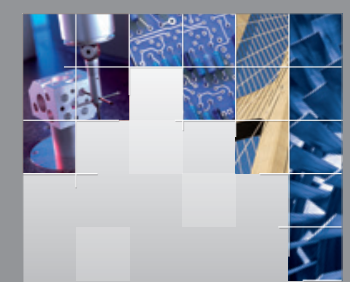

\section{Enfincering}
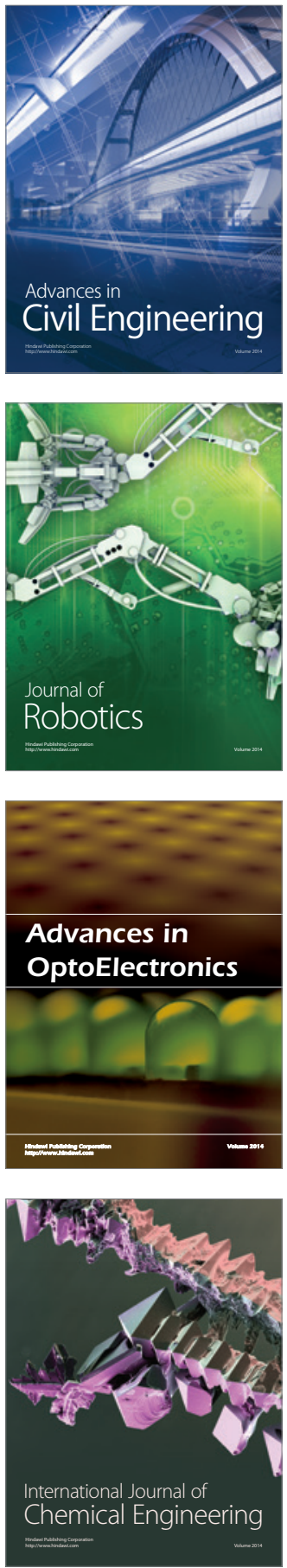

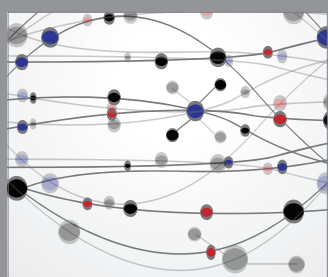

The Scientific World Journal

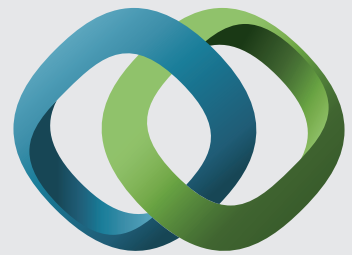

\section{Hindawi}

Submit your manuscripts at

http://www.hindawi.com
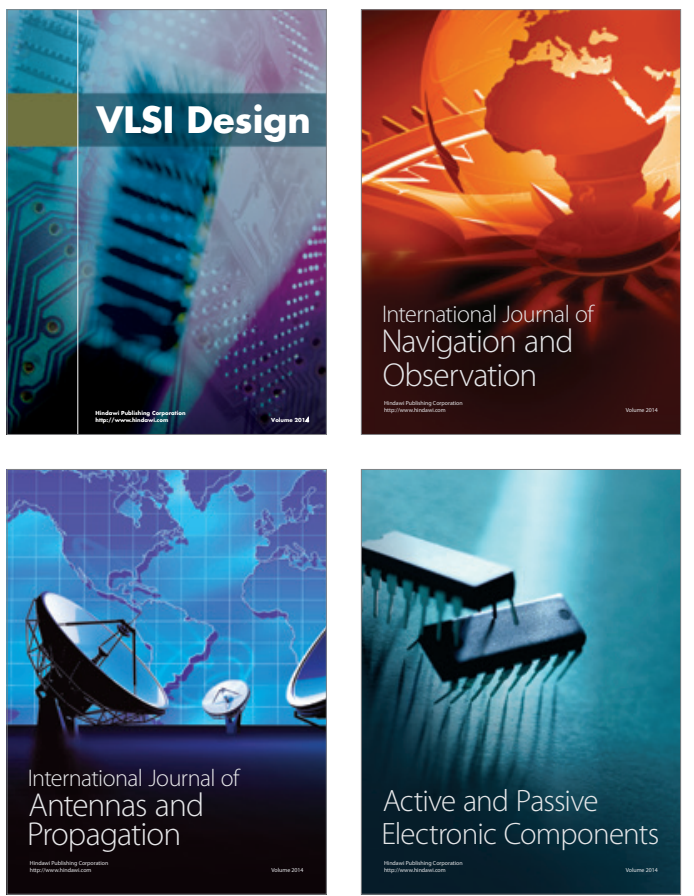
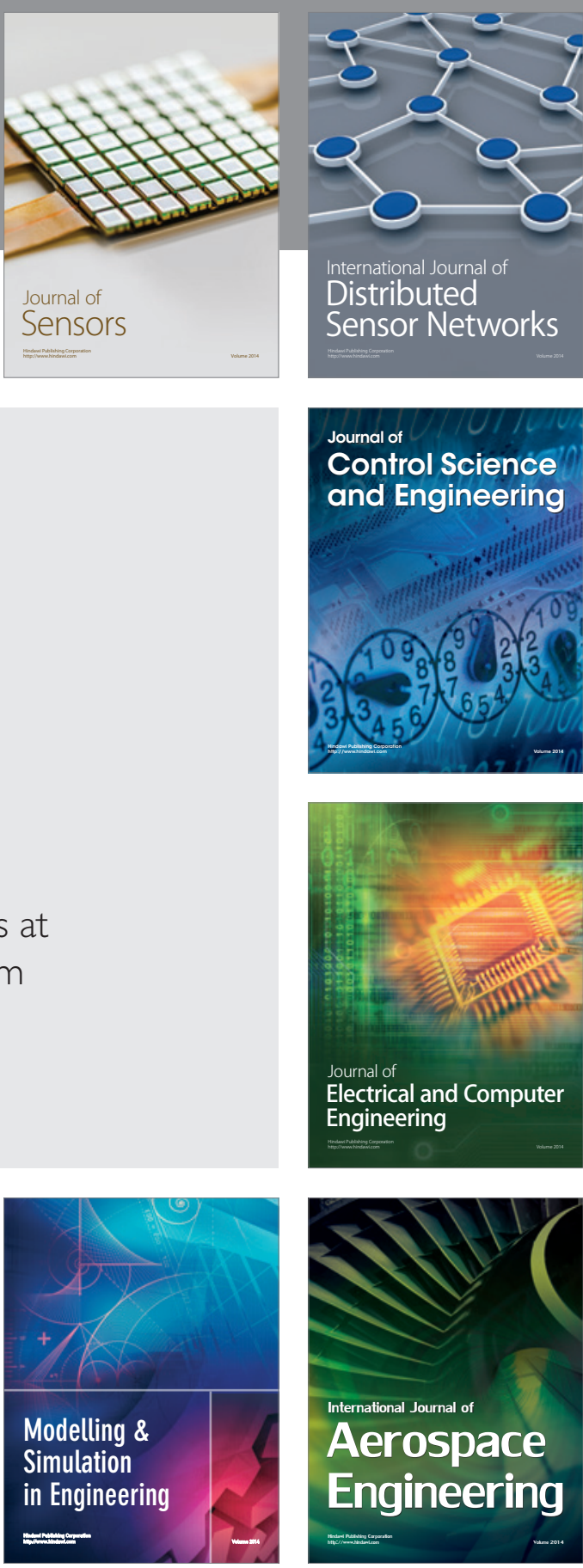

International Journal of

Distributed

Sensor Networks

Journal of

Control Science

and Engineering
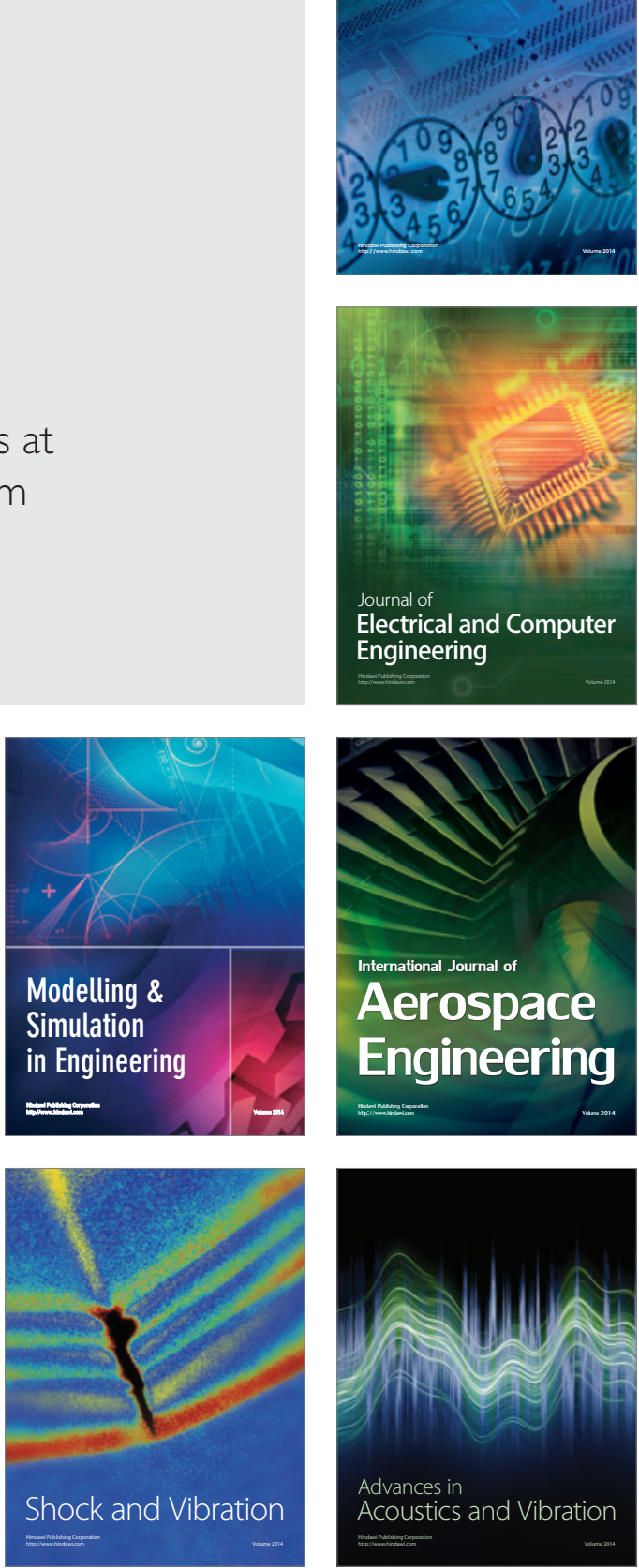